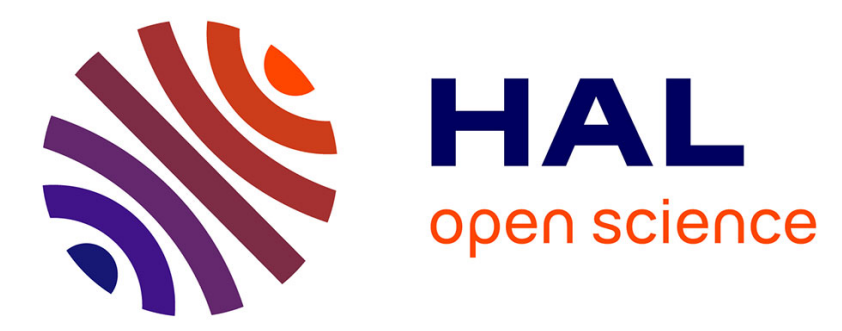

\title{
High-speed train suspension health monitoring using computational dynamics and acceleration measurements
}

David Lebel, Christian Soize, Christine Fünfschilling, Guillaume Perrin

\section{To cite this version:}

David Lebel, Christian Soize, Christine Fünfschilling, Guillaume Perrin. High-speed train suspension health monitoring using computational dynamics and acceleration measurements. Vehicle System Dynamics, 2020, 58 (6), pp.911-932. 10.1080/00423114.2019.1601744 . hal-02100228

\section{HAL Id: hal-02100228 \\ https://hal.science/hal-02100228}

Submitted on 17 Nov 2019

HAL is a multi-disciplinary open access archive for the deposit and dissemination of scientific research documents, whether they are published or not. The documents may come from teaching and research institutions in France or abroad, or from public or private research centers.
L'archive ouverte pluridisciplinaire HAL, est destinée au dépôt et à la diffusion de documents scientifiques de niveau recherche, publiés ou non, émanant des établissements d'enseignement et de recherche français ou étrangers, des laboratoires publics ou privés. 


\title{
High-speed train suspension health monitoring using computational dynamics and acceleration measurements
}

\author{
Lebel, David ${ }^{\mathrm{a}, \mathrm{b}}$, Soize, Christian ${ }^{\mathrm{a}}$, Funfschilling, Christine ${ }^{\mathrm{b}}$ and Perrin, Guillaume ${ }^{\mathrm{c}}$ \\ ${ }^{a}$ Université Paris-Est Marne-la-Vallée, MSME, UMR 8208 CNRS, 5 Bd Descartes 77454 \\ Marne-la-Vallée Cedex 2, France; \\ ${ }^{\mathrm{b}} \mathrm{SNCF}$ Innovation \& Recherche, $40 \mathrm{Av}$ des Terroirs de France, 75012 Paris, France; \\ ${ }^{\mathrm{c}} \mathrm{CEA}$ DAM DIF, Bruyères-le-Chatel, 91297 Arpajon Cedex, France
}

\begin{abstract}
This paper presents a novel method for the state health monitoring of high-speed train suspensions from in-line acceleration measurements by embedded sensors, for maintenance purposes. We propose a model-based method relying on a multibody simulation code. It performs the simultaneous identification of several suspension mechanical parameters. It is adapted to the introduction of uncertainties in the system and to the exploitation of numerous high-dimensional measurements. The novel method consists in a Bayesian calibration approach using a Gaussian process surrogate model of the likelihood function. The method has been validated on numerical experiments. We demonstrate its ability to detect evolutions of the health state of suspension elements. It has then been tested on actual acceleration measurements to study the time evolution of the suspension parameters.
\end{abstract}

\section{KEYWORDS}

railway dynamics; Bayesian calibration; state health monitoring; high-speed train suspension; surrogate model

\section{Introduction}

Trains dynamic behaviour strongly relies on suspensions that ensure the train stability and thus the ride safety. The suspensions also filter most of the vibrations for passengers comfort. In this paper, we focus on the case of passive suspensions that are used on the type of train considered in this study. Because the suspensions undergo deterioration throughout their lifetime, regular maintenance is required. Maintenance is however performed without having access to the real state of the suspensions, especially its (potentially degraded) mechanical properties. Presently, it mostly relies on visual inspection and age or mileage criteria. A monitoring solution providing the actual suspensions health state would allow for implementing maintenance rules closer to the real needs. An overwiew of modern techniques for railway vehicles on-board monitoring systems can be found for instance in [1]. The work presented here is part of a project developing a health state monitoring method for high-speed train suspensions using on-track measurements of the train dynamic response performed thanks to embedded accelerometers.

Relying solely on measurements of the train dynamic response is not sufficient, because of its strong sensitivity to the track geometry irregularities. The latter constitute the main excitation source of a rolling train and, consequently, have a major influence 


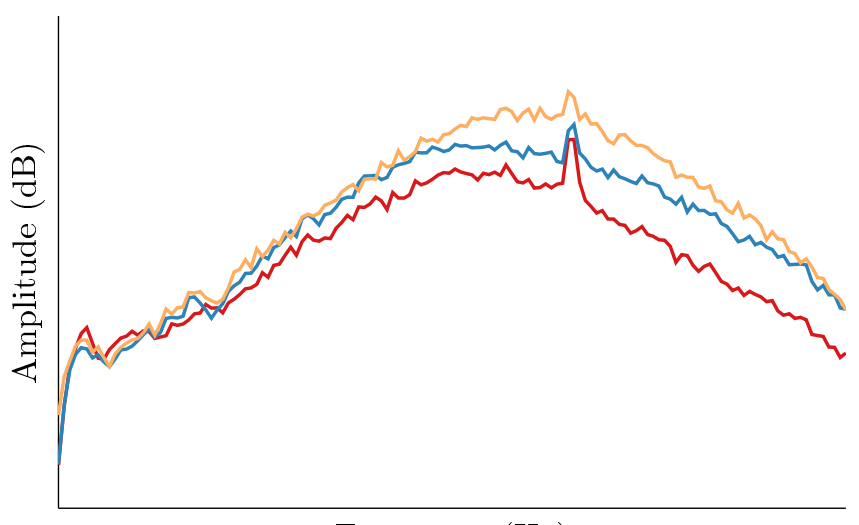

Frequency $(\mathrm{Hz})$

Figure 1. Influence of the track geometry. The three curves represent the mean value of the amplitude of the Fourier transform of a bogie vertical acceleration signal, obtained from simulation using geometry records measured at three different years.

on its dynamic behaviour (see [2-5]). Track geometry is also subject to deterioration caused by railway traffic (see [6,7]): it is gradually degraded and regularly maintained. A large quantity of data is used for the suspension monitoring, which implies that measurements performed over track geometries of various age after maintenance are available. One could expect that with a sufficiently large amount of data, the averaging effect would remove the influence of the track geometry degradation. We observed that it is not the case. We performed train dynamics simulations along the whole line considered for this work, with the exact same conditions (identical train model, same constant speed), but using track irregularities measurements performed at different years. As shown on Figure 1, the simulation revealed significant variations in the average train dynamic response from one year to another. This highlights the need of taking into account the actual track irregularities in order to perform a correct monitoring of the suspensions state. Train dynamics simulation is thus necessary to include the excitation source in the analysis. It also has the advantage to explicitly accounts for the various suspension elements through the input parameters describing their mechanical characteristics.

The proposed monitoring strategy consists of the inverse identification of the parameters of a simulation-based model. The experimental data used for the identification consists of joint measurements of the track geometry irregularities and of the train dynamic response over an entire line. This large amount of data allows for a good robustness of the identification results. In addition, the various uncertainties of the system must be accounted for to perform a robust monitoring, which results in the manipulation of random quantities of interest. Consequently, a Bayesian approach is adopted: the parameter identification problem is solved thanks to a Bayesian calibration procedure.

Relying on simulation raises a numerical cost issue that we address thanks to Gaussian process surrogate models. However, we are considering a system with functional output (the train dynamic response), which rules out the classical surrogate modelling of the simulation output. This leads us to the proposition of a novel Bayesian calibration method based on a surrogate model of the likelihood function.

In this paper, the focus is set on the industrial problem and on the results obtained with the proposed suspension health state monitoring method. The mathematical 
aspects of the method are not detailed. To this end, we refer to [8,9]. A short review of the existing method for train suspension monitoring using acceleration measurements is proposed in Section 2. Details about the train and the suspension elements of interest are given in Section 3. Section 4 presents the Bayesian calibration while Section 5 presents the main aspect of the proposed novel method. Finally, results are analysed in Section 6.

\section{State of the art}

During the last decades, various methods have been proposed for the health state monitoring of train suspensions. They rely on the measurements provided by embedded accelerometers. The literature focuses on the mathematical processing of the dynamic response signals, which allows for extracting information about the train suspension elements.

The preliminary tests presented in [10] demonstrate the potential of accelerometers for this purpose. Because the suspension elements affect the dynamic behaviour of the rolling train, it is assumed that, reciprocally, observing this behaviour can provide information about the state of these suspensions. In [10], the authors underline the interest of the analysis of the acceleration signals in both time and frequency domains.

Some important distinctions can be made between the methods listed in the following sections. The first distinction concerns the monitoring objective: the detection of a sudden fault in one of the suspension elements or the study of their gradual degradation or wear, if they are subject to the latter. Fault detection usually considers bigger changes in the suspensions mechanical characteristics. The fault detection methods work on short time scales to allow for a quick action after the fault happens. On the contrary, the monitoring of wear is performed on a longer time-scale but may look for smaller evolution of the suspensions health state. The literature (see the following references) focuses mostly on sudden fault detection, even though it can be argued that a fault detection method may be applied to the study of suspensions wear.

The second distinction to be made is between fault detection, fault isolation, and fault magnitude quantification. Fault detection is a first step that consists in observing a change in the train behaviour that is considered to be the consequence of a fault happening. Fault isolation is the subsequent step that consists in identifying the faulty suspension element. Fault magnitude quantification consists in estimating the value of the faulty suspension mechanical characteristics to determine the damage level. Most solutions dealing with sudden suspension fault focus on fault detection and isolation, often with no clear separation between these two steps.

The third distinction concerns the need of a railway dynamics model. They can provide precious insight about the relationship between the suspensions state and the train dynamic behaviour. They require the construction of an accurate vehicle model, in which the suspension elements are explicitly represented and associated with mechanical parameters. Most health state monitoring methods developed lately rely on simulation at some point. Therefore, the quality and representativity of railway dynamics models is of high importance for monitoring purposes. Three different categories of methods can be considered:

- the methods that rely only on the measured data and do not require any model. Because they are appropriate for fault detection only, such methods are not considered in the present paper. Refer for instance to [11]; 
- the fault identification methods that do not need a train model for the monitoring procedure itself, but require a prior training. The latter is usually achieved thanks to simulation results, but ideally measured data should be used. These methods are designated as data-driven methods and developed in Section 2.1.

- the fault identification methods where the dynamic model is at the core of the monitoring procedure, often through the use of Kalman filters. These methods are designated as model-based methods and developed in Section 2.2.

\subsection{Data-driven methods in the context of train suspension monitoring}

In [12], the authors propose a fault detection method relying on the analysis of the cross-correlation function between different body motions. For vehicles with symmetrical design, they show that the coupling between the different motions is small. A faulty element alters the symmetry, which results in a coupling between motions that can be observed in the cross-correlation function. Focusing on the vertical primary suspension elements, they highlighted the impact of a faulty damper on the crosscorrelations between the bounce, pitch and roll acceleration signals. In the studied case, fault isolation seems possible but requires that each type of fault be associated with its effect on the cross-correlation signals.

In [13], the authors perform a experimental modal identification of the train behaviour from the acceleration measurements. The frequency and damping ratio of several modes are identified. Thanks to simulation results using the multi-body code Vampire $\AA$, regression models are identified between the frequency, the damping ratio and the value of the parameter describing the suspension state, for several modes and various degradation configurations. The regression can then be used in inverse to determine the suspension state from frequencies and damping ratio estimated from experimental modal identification.

The method proposed in [14] is also based on a frequency analysis of the acceleration signal. The Random Decrement Technique (RDT) provides an approximation of the signal auto-correlation. The decomposition in Prony series then allows for estimating the natural frequencies and damping ratios of the vehicle free oscillations. The mean and standard deviation of the first natural frequency and damping ratio on various acceleration samples are then used to determine the fault type and a level of magnitude thanks to a k-NN (k Nearest Neighbours) categorization method. The k-NN initial training is achieved thanks to simulation results generated using a multi-body software.

In [15], a baseline Autoregressive with exogenous excitation (ARX) model is identified from experimental data. It represents the relation between the system input (the track excitation) and response (the accelerations) as an IIR filter with a white noise residual. On this baseline model structure a functional ARX (FARX) model is identified. It represents a faulty train, with coefficients depending on the fault magnitude. Only one type of suspension fault can be represented by a given FARX model. The FARX identification is performed thanks to simulation results. The fault detection and identification procedure works as follows: the measured accelerations are injected in the FARX model; the magnitude of the fault is determined by minimizing the FARX residual variance. When this fault magnitude is above a threshold, the fault type is validated if the residual is sufficiently uncorrelated. 


\subsection{Model-based methods in the context of train suspension monitoring}

Most model-based method rely on Kalman filters to perform a state health monitoring of train suspensions. The purpose of linear Kalman filtering is to follow the timeevolution of a dynamic system using a linear model describing the system behaviour while taking into account the information provided by regular measurements of some observable quantities. A basic linear Kalman filter can be described thanks to two equations:

$$
\begin{aligned}
\mathbf{X}_{k+1} & =[A] \mathbf{X}_{k}+\mathbf{U} \\
\mathbf{Y}_{k} & =[H] \mathbf{X}_{k}+\mathbf{V}
\end{aligned}
$$

where $\mathbf{X}_{k}$ and $\mathbf{Y}_{k}$ are the state-space vector and the observation vector at step $k$, $[A]$ the state-transition matrix, $[H]$ the observation matrix, $\mathbf{U}$ and $\mathbf{V}$ are independent centred Gaussian vectors representing the process noise and the observation noise. At each time step $k$, the state-space vector is updated using Eq. (1) and then conditioned by the available experimental observation using Eq. (2). In the case of railway dynamics, state-space vector $\mathbf{X}_{k}$ usually gathers the degrees of freedom of the various masses constituting the vehicle and their derivatives, while observation vector $\mathbf{Y}_{k}$ consists of the accelerations measured in different locations. The suspensions mechanical parameters are used to compute matrices $[A]$ and $[H]$. A Kalman filter is designed to estimate the time-evolution of the state-space variables. On the contrary, the parameters are supposed to be fixed and given. Adaptations are thus required in order to perform Kalman-based condition monitoring.

A first approach described in [16] is to consider the parameters of interest as statespace variables. An evolution equation must be defined for the parameters. Moreover, using an augmented state-space vector makes the system non-linear, which is solved by using an Extended Kalman Filter (EKF). EKF uses the Jacobian matrix of the system to perform a linearisation around the current value of the state-space vector. However, the simulataneous estimation of several parameters using EKF appears to achieve bad results. Consequently, in [16] they propose to use multiple EKF in parallel, each one of them performing the identification of a single suspension parameter. A modification of the train response can lead to changes in the estimated value of several parameters. The most probable faulty suspension is then identified from a likelihood estimation of the measurements with respect to the various faulty models.

Several other papers propose to use multiple Kalman filters in parallel. In [17], multiple Kalman filters are run in parallel, each one modelling a fault type, with fixed magnitude. It means that the state-transition and the observation matrices are modified for each filter to take into account the degraded suspensions parameters. The fault identification is once again performed from a likelihood estimation (averaged over several time-steps) of the measurements with respect to the different models.

The IMM (Interacting Multiple Models) algorithm presented in [18] is similar but additionally includes mode mixing. The parallel Kalman filters are no longer isolated but interact with one another. The input state-space vector at each time step for a given filter is a combination of the output state-space vector of all filters at the previous time step. This combination is based on the modes likelihood and on given transition probabilities between modes. In a subsequent paper [19], the authors propose a model updating procedure to adapt the baseline model as a fault is detected and to allow for the identification of simultaneous faults. The idea is to work with several groups of Kalman filters in parallel, each one focusing on one type of suspension. The algorithm 
allows a modification in the suspensions parameters detected by one group to update the models of the other groups.

The method proposed in [20,21], although relying on multiple Kalman filters, differs from the previous ones because the associated models are not chosen in advance to represent fixed fault types and magnitudes. In a similar fashion to EKF-based parameter estimation, the aim is to estimate the probability distribution of an augmented state-space vector gathering the initial state-space variables (the degrees of freedom of the multi-body model) and the suspension parameters. The authors then propose to separate the estimation of the probability distributions of the state-space variables and of the parameters thanks to marginalization. A Rao-Blackwellised particle filter is used to represent the probability distribution of the parameters by a weighted sample, while linear Kalman filters are used to estimate the state-space variables.

The fault identification method proposed in [22] relies on a single EKF, for the case when the various degrees of freedom are decoupled. More precisely, the degrees of freedom associated with one suspension of interest must not be linked to other suspension elements. The use of EKF allows for considering dampers with non-linear properties. The fault identification procedure focuses on the Kalman filter innovation. Its norm is minimized by adding an error to the degrees of freedom associated with a suspension of interest. The magnitude of this error determines whether the corresponding suspension must be considered as faulty.

In [23], the authors also focus on the Kalman filter innovation. A fault is detected when the weighted sum squared residual (averaged over several time steps), equivalent to the log-likelihood of the observations, crosses a given threshold. A possible fault identification procedure is then suggested, which consists in the analysis of the norm or the power spectral density of the different component of the Kalman innovation.

In [24], no Kalman filter is used, but instead a closely related time-domain filter known as Recursive Least Squares (RLS). RLS is an algorithm able to identify the parameters of an input-output linear system by filtering the error signal between the measured and modelled outputs. It requires measurements of both the input and the output signals. In this paper, the acceleration of the wheelsets is used as input, so the track irregularities do not need to be measured and included in the model.

Most of the cited articles rely on Kalman filters to propose a fault detection and isolation method for abrupt changes in the suspensions characteristics. This is underlined by the magnitudes of the fault that are considered (often $50 \%$ or 100\%). Considering that this type of failures represent a risk for the ride safety, these methods mostly focus on a quick detection and exploit acceleration signals measured over a few seconds.

In the present paper, we are interested in the long-time degradation of the suspension elements. We exploit large measurements data-bases, corresponding to rides along several hundreds of kilometres of track, to ensure the robustness of the monitoring results.

In the cited articles, the train models usually consist of a single trailer, and consider only certain degree of freedom and the associated suspension elements, for which in general, the non-linearities are not taken into account. On the contrary, the modelbased approach used in this paper includes the full representation of an entire train, including non-linearities for wheel-rail contact and in suspension mechanical characteristics. It allows for the simultaneous monitoring of several suspensions elements, with or without strong couplings. This requires simulations using a commercial nonlinear multibody code, Vampire ( $)$. The modelling is thus more physical and allows for finer identifications.

In the cited articles, the inclusion of the various uncertainties of the system is often 


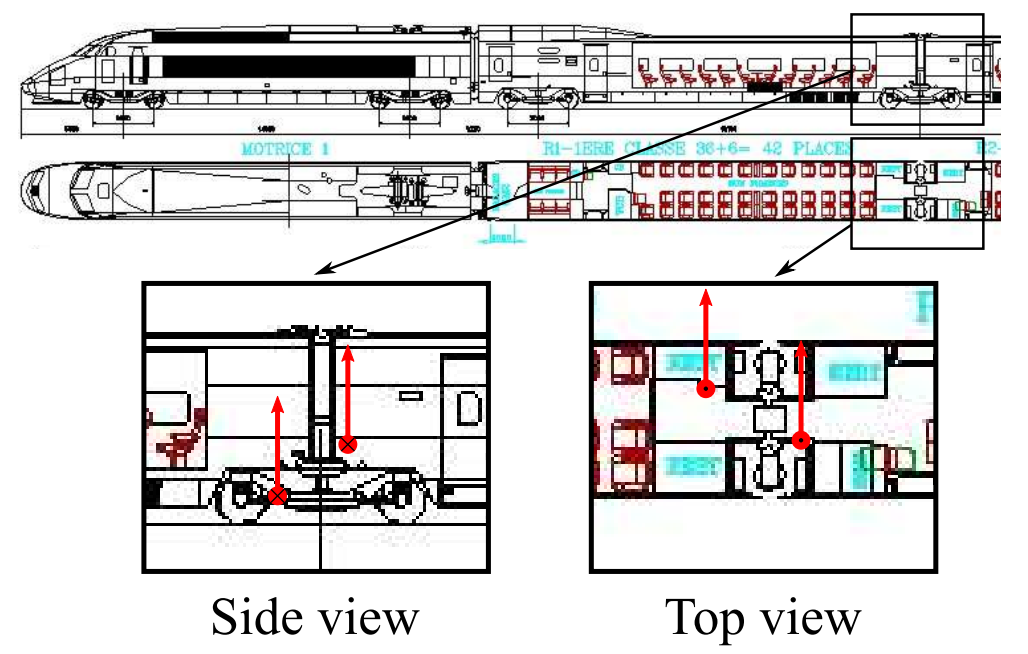

Figure 2. Location of the two accelerometers on the train carbody and bogie, and axes directions in which the accelerations are measured.

lacking. Even though Kalman filters naturally take measurement noise into account, no model error is considered in general. The necessity to include the various uncertainties of the system motivates our choice to rely on a Bayesian approach. Many papers model the track geometry irregularities as a simple coloured random noise. As explained in the introduction, we are convinced of the importance of using the real geometry whenever possible. For this work, actual measurements of the track geometry irregularities are used instead of considering them as a basic random input.

Finally, certain of the proposed method are tested on numerical experiments but rarely on actual acceleration measurements. In this paper, we presents the results of the application of the method on several sets of measurements.

\section{Studied system}

In this paper, the studied vehicle is a French TGV Réseau, an articulated one-level high-speed train with passive suspensions. Articulated means that the bogies are shared between two carbodies, except for the two motor cars at each end of the train.

\subsection{Available data}

Two carbody junctions are studied: the first between the two first trailers, the second between the two last trailers. For each junction, one sensor is located on a carbody, and one on the bogie (see Figure 2). They measure both vertical and lateral accelerations. Since the sensors location do not coincide with the centres of mass, the measured accelerations correspond to a combination of the different body motions. In addition to these eight acceleration signals, the location of the train along the track as well as its speed are recorded.

Measurements of the track geometry irregularities corresponding to each circulation are available. They consist of four signals representing the vertical, lateral, cross-level, and gauge irregularities. The track curvature has a strong impact on both the train dynamic behaviour and the irregularities. Consequently, the track has been divided 


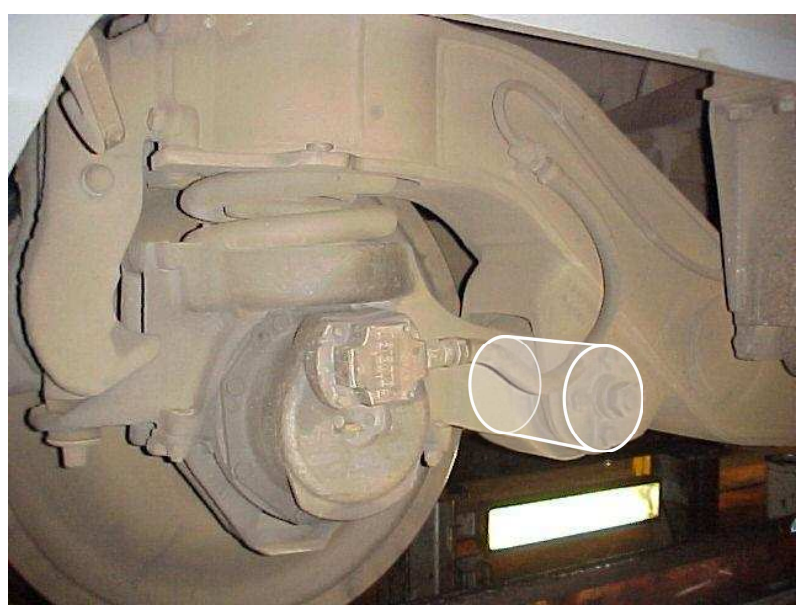

Figure 3. Picture of the train primary suspension. The location of the elastomeric stiffness is sketched as a white cylinder.

into various stretches depending on the curvature in order to gather more statistically coherent datasets.

\subsection{Suspension elements of interest}

The suspension elements of interest are: the elastomeric stiffness (primary suspension), the airspring (secondary suspension), the vertical primary damper, the yaw damper, and the upper inter-trailer damper. This selection has been determined based on the needs expressed by the maintenance workshops: we focused on the elements involved in the train safety, requiring frequent inspection or inducing high maintenance costs. Moreover, we ruled out those whose influence on the accelerations measured by the sensors is to small (based on simulation results). It should be noted that another sensors configuration may be more sensitive to the degradation of these elements. From the initial sensitivity analysis, we also figured out that with the given sensors configuration, we were not able to locate a fault on a specific element among a set of elements of the same type. Consequently, for each junction, we choose to make all the elements of a same type depend on common parameters. Reducing the number of parameters also decreases the problem dimension.

The admissible set for each parameter is defined from the specifications detailed in the vehicle blueprints, as an interval centred around the nominal value. Since the objective is to monitor potentially degraded suspensions, the interval width is set to stretched specification margins.

\subsubsection{Elastomeric stiffness}

On the studied bogie, the motion of the wheelset with respect to the bogie frame is constrained by trailing arms, as shown in Figure 3. The connection between these arms and the bogie frame is ensured by an axial joint containing an elastomeric stiffness. This stiffness works in torsion and is linked to the vertical motion of the wheelset. It comes in addition to the primary vertical coil spring (which are not considered in the parameter identification).

Elastomers are hyperelastic viscoelastic materials with a complex mechanical be- 


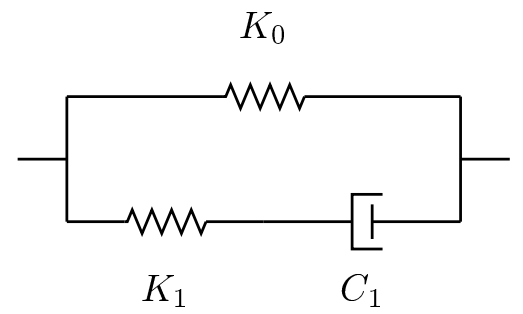

Figure 4. Rheological model for the elastomeric stiffness.

haviour. The stiffness usually depends on the strain, the solicitation frequency, the temperature, etc. In order to keep a simple train model and a light parametrization, the elastomeric stiffness is modelled by a linear rheological model (similar to a Zener model) consisting of a spring in parallel with a branch made of a damper and a spring in series, as represented in Figure 4. This model allows for representing, in a simplified way, the softening of the material at low frequencies. Stiffness $K_{0}$ is considered as the parameter to identify, while $K_{1}$ and $C_{1}$ are determined from $K_{0}$ according to the recommendations given for the use of Vampire $\AA$.

\subsubsection{Airspring}

Airsprings are nowadays a very common secondary suspension type for passenger trains. Their ability to filter vibrations plays an important role in the ride comfort. Basically, an airspring consists of an elastomeric membrane under pressure supporting the vertical load of the carbody, linked by a surge pipe to a reservoir. They are mainly involved in the vertical motion (and consequently in the roll and pitch motions) of the carbody through pneumatic effect. They also affect the lateral motion of the carbody through the shear strain of the elastomeric membranes.

Various rheological models of airsprings can be found in the literature (see for instance [25]). They usually require tuning numerous parameters in order to reproduce the complete behaviour of this type of suspension. We have chosen to rely on a much simpler model that represents the airspring as constant stiffnesses and dampers in parallel for the six degrees of freedom between the carbody and the bogie. Only the vertical damping coefficient and stiffness are considered for the parameter identification, all the others being fixed.

\subsubsection{Dampers}

Train suspension systems are equipped with numerous dampers. In this study, we focus on the vertical primary dampers, the yaw dampers, and the upper inter-trailer dampers. For railway systems, dampers usually have a non-linear mechanical behaviour, with a damping rate decreasing with velocity. Figure 5 provides an example of a damper non-linear characteristic. The latter is often approximated by a bilinear curve.

In order to limit the number of parameters to identify, the parameter for each type of damper is defined as a multiplicative factor applied to the given nominal non-linear characteristics. 


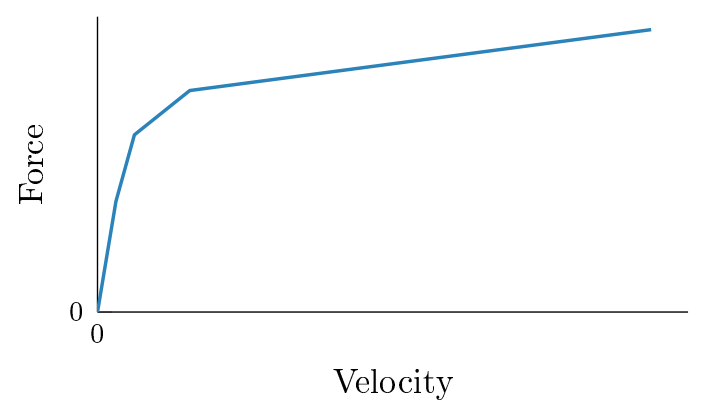

Figure 5. Example of non-linear damper characteristic

\section{Computational model-based monitoring in a Bayesian framework}

The parameter identification method chosen in the paper is Bayesian calibration. The principle is to confront a model of the system response to experimental data in order to provide information about the system parameters. The Bayesian framework allows for taking into account the system uncertainties, detailed in Section 4.1. The calibration procedure is then explained is Section 4.2. All the quantities represented by function or stochastic process depending on time, or frequency, or space, are discretised and are then represented by vectors.

\subsection{System uncertainties}

Various sources of uncertainties must be considered to perform the parameter identification.

First, the values of parameters of interest that must be identified are uncertain by definition. Each parameter is thus represented by a random variable associated. Its prior probability density function (PDF) represents the initial knowledge available about this random variable, which may be deduced from specifications, tests or experts judgements. In the present case, because of the lack of initial knowledge about the parameters, the prior PDFs are chosen as uniform distributions on the parameters admissible intervals. It is assumed that the random variables that model the parameters are independent for the prior model and will be a priori dependent for the posterior one. The goal of Bayesian calibration is to update the prior PDF to determine the posterior PDF that takes into account the information provided by the experimental data. The parameters of interest are gathered in the random vector $\mathbf{W}$. The prior and posterior PDF of $\mathbf{W}$ are respectively denoted as $p_{\mathbf{W}}^{\text {prior }}$ and $p_{\mathbf{W}}^{\text {post }}$. The support of $p_{\mathbf{W}}^{\text {prior }}$ is the set $\mathcal{C}_{\mathbf{W}}$ that is the set product of the parameters admissible intervals.

The measurements may also be subject to uncertainties. The measured response $\mathbf{Y}^{\text {mes }}$ is written as

$$
\mathbf{Y}^{\mathrm{mes}}=\mathbf{Y}^{\mathrm{real}}+\varepsilon^{\mathrm{mes}}
$$

where $\mathbf{Y}^{\text {real }}$ is the 'real' physical response and $\varepsilon^{\text {mes }}$ is a random quantity representing the measurement uncertainties.

No model can perfectly represent a physical system. It may be affected by various errors, such as: uncertainties on model parameters, simplification errors introduced 


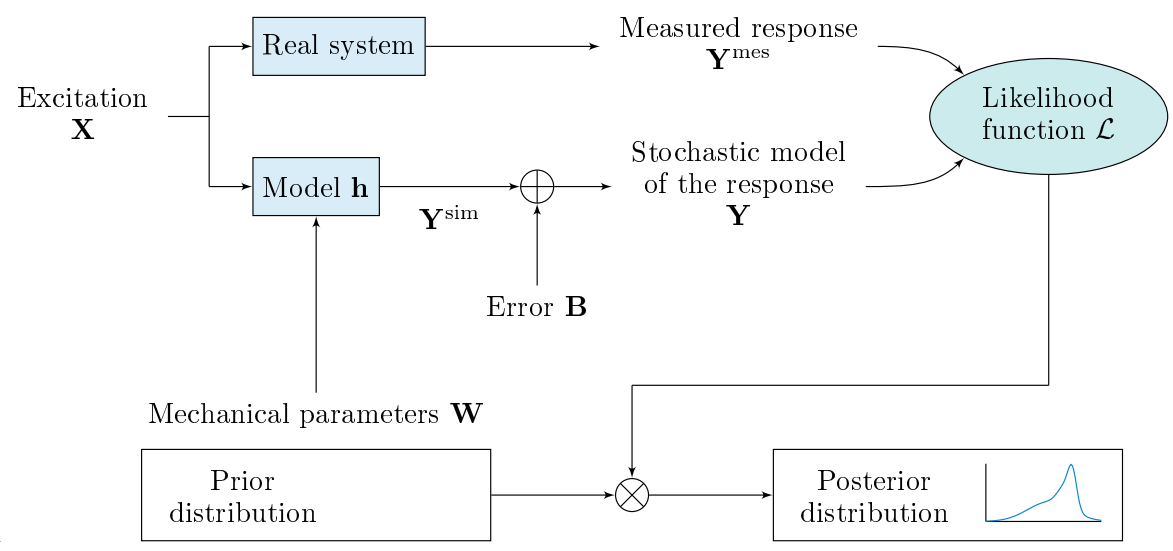

Figure 6. Diagram of the Bayesian calibration method

in the construction of the model, discretisation errors, etc. They are globally taken into account thanks to a random model error that represents the distance between the model response and the 'real' physical response:

$$
\mathbf{Y}^{\text {sim }}=\mathbf{Y}^{\text {real }}+\varepsilon^{\text {sim }}
$$

where $\mathbf{Y}^{\text {sim }}$ is the model response and $\varepsilon^{\text {sim }}$ is the model error. Because we are not able to isolate the measurement uncertainties and the model error, they are taken into account globally thanks to an output predictive error $\mathbf{B}$ representing the distance between the measured and the model response. We define the stochastic model of the system response as

$$
\mathbf{Y}=\mathbf{Y}^{\mathrm{sim}}-\varepsilon^{\mathrm{sim}}+\varepsilon^{\mathrm{mes}}=\mathbf{Y}^{\mathrm{sim}}+\mathbf{B}
$$

By construction, random variable $\mathbf{Y}$ is assumed to model measured response $\mathbf{Y}^{\text {mes }}$. In fact, the Bayesian calibration procedure consists in 'fitting' $\mathbf{Y}$ to $\mathbf{Y}^{\text {mes }}$ that is only known through a set of realisations. Error B is identified as a Gaussian vector from measurements performed at a reference date, defined as the date for which the values of the parameters of interest are known. In the present case, the reference date is chosen as the date immediately following a major maintenance operation. At the reference date, the parameters are thus supposed to be nominal.

\subsection{Bayesian calibration principle}

The procedure is sketched in Figure 6. A model-based parameter identification is performed. The procedure is thus built around the train dynamics simulation code Vampire (B. It is a deterministic multibody non-linear dynamics simulation code, working in the time domain.

The input excitation of the system consists of the track geometry irregularities represented by the stochastic process $\mathbf{X}$. They can be modeled by the non-stationary stochastic process, presented in [26]. Since the track irregularities are simultaneously measured with the train dynamic responses, we choose to consider these measurements on various track stretch as a set of realisations of this stochastic process.

The simulation output consists of acceleration signals in the time domain corre- 
sponding to those measured by the embedded accelerometers. In order to avoid systematic phase shift between the measured and simulated signals, they are transformed into the frequency domain. The model response $\mathbf{Y}^{\text {sim }}$ then consists in the amplitude (in $\mathrm{dB}$ ) of the Fourier transform of the acceleration signals. As explained in the previous section, an output predictive error $\mathbf{B}$ is then added to the model response $\mathbf{Y}^{\text {sim }}$ in order to obtain the stochastic model of the train dynamic response $\mathbf{Y}$. Error $\mathbf{B}$ is supposed to be independent from input $\mathbf{X}$ and parameters $\mathbf{W}$.

Using the stochastic model and the experimental data, the Bayesian calibration objective is to update the PDF of the parameters of interest. It consists in estimating the posterior PDF of the parameters by conditioning the prior PDF by the measurements. We first consider the case of a single measurement. For $\mathbf{w}$ in admissible set $\mathcal{C}_{\mathbf{W}}$, using the Bayes formula, the posterior PDF is written as

$$
\begin{aligned}
& p_{\mathbf{W}}^{\text {post }}(\mathbf{w})=p_{\mathbf{W} \mid \mathbf{X}, \mathbf{Y}}\left(\mathbf{w} \mid \mathbf{x}^{\text {mes }}, \mathbf{y}^{\text {mes }}\right) \\
& \propto p_{\mathbf{Y} \mid \mathbf{W}, \mathbf{X}}\left(\mathbf{y}^{\text {mes }} \mid \mathbf{w}, \mathbf{x}^{\text {mes }}\right) p_{\mathbf{W} \mid \mathbf{X}}\left(\mathbf{w} \mid \mathbf{x}^{\text {mes }}\right) \\
& \propto \mathcal{L}(\mathbf{w}) p_{\mathbf{W}}^{\text {prior }}(\mathbf{w}),
\end{aligned}
$$

in which $\left(\mathbf{x}^{\text {mes }}, \mathbf{y}^{\text {mes }}\right)$ is the joint measurement of the track geometry irregularities and of the corresponding train dynamic response on a given track stretch, where $p_{\mathbf{W}}^{\text {prior }}(\mathbf{w})=p_{\mathbf{W} \mid \mathbf{X}}\left(\mathbf{w} \mid \mathbf{x}^{\text {mes }}\right)$ because the prior model of $\mathbf{W}$ is independent of $\mathbf{X}$, and where the likelihood function $\mathcal{L}$ is such that

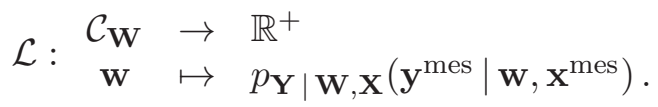

In fact, the probability distribution of the parameters is conditioned by several independent couples $\left\{\left(\mathbf{x}^{\text {mes }, i}, \mathbf{y}^{\text {mes }, i}\right)\right\}_{1 \leq i \leq \nu}$ corresponding to measurements performed on $\nu \geq 1$ track stretches. For $\mathbf{w} \in \mathcal{C}_{\mathbf{W}}$ the likelihood function is then equal to the product

$$
\mathcal{L}(\mathbf{w})=\prod_{i=1}^{\nu} p_{\mathbf{Y} \mid \mathbf{W}, \mathbf{X}}\left(\mathbf{y}^{\text {mes }, i} \mid \mathbf{w}, \mathbf{x}^{\text {mes }, i}\right) .
$$

Assuming that the likelihood function $\mathcal{L}(\mathbf{w})$ can be computed for any $\mathbf{w} \in \mathcal{C}_{\mathbf{W}}$, the following step is to apply Markov chain Monte Carlo (MCMC) to sample values of $\mathbf{W}$ whose PDF $p_{\mathbf{W}}^{\text {post }}$ is defined by Eq. (8). From this sample, the moments of $p_{\mathbf{W}}^{\text {post }}$ can be estimated. It could also be represented using kernel-density estimation methods [27].

To conclude, the critical point of the Bayesian calibration procedure lies in the computation of the likelihood function. In general, it depends on the nature of the stochastic model Y. In the present case, the computational can be represented by the deterministic mapping $\mathbf{h}$ such that

$$
\begin{gathered}
\mathbf{Y}^{\operatorname{sim}}=\mathbf{h}(\mathbf{X} ; \mathbf{W}) \\
\{\mathbf{Y} \mid \mathbf{W}=\mathbf{w}, \mathbf{X}=\mathbf{x}\}=\mathbf{h}(\mathbf{x} ; \mathbf{w})+\mathbf{B} .
\end{gathered}
$$

In the right-handside of Eq. (12), only $\mathbf{B}$ is random, the expression of the likelihood 
function defined by Eq. (10) can then be rewritten as

$$
\mathcal{L}(\mathbf{w})=\prod_{i=1}^{\nu} p_{\mathbf{B}}\left(\mathbf{y}^{\mathrm{mes}, i}-\mathbf{h}\left(\mathbf{x}^{\mathrm{mes}, i} ; \mathbf{w}\right)\right), \mathbf{w} \in \mathcal{C}_{\mathbf{W}} .
$$

Error $\mathbf{B}$ is identified as a Gaussian vector, which makes its PDF $p_{\mathbf{B}}$ easy to represent. However, Eq. (13) shows that $\nu$ calls to the computational model represented by mapping $\mathbf{h}$ are required to compute the value of the likelihood $\mathcal{L}$ for one value of $\mathbf{w}$. The simulation being expensive makes each call to the likelihood function expensive. In addition, MCMC usually requires numerous calls to the likelihood function to generate a representative sample of the posterior PDF.

A solution to circumvent such numerical cost issue is to resort to surrogate models. Bayesian calibration using GP surrogate models has already been studied when the system response is scalar. In that case, the classical method is to build a surrogate model of the simulation output. The uncertainties induced by the introduction of a surrogate model can then be directly taken into account in the calculation of the likelihood function. However, in the present case, we are dealing with functional outputs: the amplitude of the Fourier transform of the acceleration signals. The surrogate model of a functional quantity is much more difficult to build. Numerous calls to such a surrogate model are also necessary to aggregate the value of likelihood function, which may affect the method efficiency. It remains a subject of current research. In this paper, we do not use the classical approach consisting in constructing a surrogate model of mapping $\mathbf{h}$ but a novel approach consisting in constructing a surrogate model of the likelihood function to perform the Bayesian calibration.

\section{Novel Bayesian calibration method using a surrogate model of the likelihood function}

The estimation of the posterior PDF of $\mathbf{W}$ requires numerous calls to the likelihood function. Each call requires a call to the computational model. The proposed strategy is to compute the likelihood values on an initial training set, and to use this information to build a surrogate model approximating efficiently the likelihood function anywhere in its definition set. In fact, we make the common choice of working with the loglikelihood function denoted as $L$ instead of the likelihood function $\mathcal{L}$.

Gaussian process surrogate modelling consists in identifying a Gaussian process indexed on the admissible set $\mathcal{C}_{\mathbf{W}}$ approximating the log-likelihood function. The real unknown log-likelihood function is supposed to be a particular realisation of this stochastic process. The GP surrogate model of $L($.$) is denoted as L(. ; \Theta)$ in which $\Theta$ is a random variable modeling the uncertainty induced by the introduction of the surrogate model. At any point $\mathbf{w} \in \mathcal{C}_{\mathbf{W}}$, the Kriging predictor is denoted as the expectation $E_{\Theta}\{L(\mathbf{w} ; \Theta)\}$ and constitutes the best approximation of $L(\mathbf{w})$ in the mean-square sense. The variance $\operatorname{Var}_{\Theta}\{L(\mathbf{w} ; \Theta)\}$ quantifies the approximation error of the Kriging predictor. If $\theta$ is a realisation of $\Theta$, then $L(. ; \theta)$ represents a deterministic trajectory of the GP model.

MCMC can be applied to a likelihood function (a conditional PDF). The GP surrogate model being random, it cannot directly be used as such. To perform a sampling of the posterior PDF with MCMC, the most straightforward solution is to use the Kriging predictor $E_{\Theta}\{L(. ; \Theta)\}$ instead of $L($.$) . We refer to this approach as the KP$ 
(for 'Kriging predictor') method. Once the GP model is built, this solution is easy to implement. It can provide useful results, especially if only the most probable values or the mean values of the system parameters are needed (see the results in Section 6.2).

However, this solution does completely ignore the uncertainty introduced by the use of a surrogate model, which represents the approximation error intrinsic in such modelling. The uncertainty on the calibrated parameters would then only be due to the uncertainties of the system represented by random vector $\mathbf{B}$. We thus propose a method that takes into account the uncertainty of the GP surrogate model in order to estimate more correctly the calibration error. We refer to this new approach as the MCT (for 'Monte Carlo on the trajectories') method that is defined hereinafter.

If the deterministic log-likelihood function $L($.$) is replaced by the random surrogate$ model $L(. ; \Theta)$, the corresponding posterior PDF becomes random as well. The random posterior PDF corresponding to $L(. ; \Theta)$ can be expressed using the conditioning by $\Theta$ as

$$
\mathbf{w} \mapsto p_{\mathbf{W} \mid \mathbf{X}, \mathbf{Y}, \Theta}\left(\mathbf{w} \mid \mathbf{x}^{\text {mes }}, \mathbf{y}^{\text {mes }}, \Theta\right) .
$$

However, the quantity we are looking for remains unchanged: the conditional PDF of parameters $\mathbf{W}$ knowing $(\mathbf{X}, \mathbf{Y})$. The rule of conditional probabilities states that it is equal to the expectation with respect to $\Theta$ of the previous PDF:

$$
\begin{aligned}
p_{\mathbf{W}}^{\text {post }}(\mathbf{w}) & =p_{\mathbf{W} \mid \mathbf{X}, \mathbf{Y}}\left(\mathbf{w} \mid \mathbf{x}^{\text {mes }}, \mathbf{y}^{\text {mes }}\right) \\
& =E_{\Theta}\left\{p_{\mathbf{W} \mid \mathbf{X}, \mathbf{Y}, \Theta}\left(\mathbf{w} \mid \mathbf{x}^{\text {mes }}, \mathbf{y}^{\text {mes }}, \Theta\right)\right\} \\
& \approx \frac{1}{N} \sum_{j=1}^{N} p_{\mathbf{W} \mid \mathbf{X}, \mathbf{Y}, \Theta}\left(\mathbf{w} \mid \mathbf{x}^{\text {mes }}, \mathbf{y}^{\text {mes }}, \theta_{j}\right) .
\end{aligned}
$$

Equation (17) corresponds to the empirical estimate of the expectation using $N$ independent realisations $\left\{\theta_{j}\right\}_{1 \leq j \leq N}$ of random variable $\Theta$. This Monte Carlo approach requires the sampling of $N$ PDFs $p_{\mathbf{W} \mid \mathbf{X}, \mathbf{Y}, \Theta}\left(\mathbf{w} \mid \mathbf{x}^{\text {mes }}, \mathbf{y}^{\text {mes }}, \theta_{j}\right)$ by MCMC using $N$ deterministic trajectories $L\left(. ; \theta_{j}\right)$ of surrogate model $L(. ; \Theta)$. In practice, for each $j$, the MCMC algorithm provides a sample of $\mathbf{W} \mid \Theta=\theta_{j}$ that is subset of $\mathcal{C}_{\mathbf{W}}$, which is distributed as $p_{\mathbf{W} \mid \mathbf{X}, \mathbf{Y}, \Theta}\left(\mathbf{w} \mid \mathbf{x}^{\text {mes }}, \mathbf{y}^{\text {mes }}, \theta_{j}\right)$. The simple concatenation of these $N$ samples corresponds to a sample distributed as $p_{\mathbf{W}}^{\text {post }}$. More details can be found in $[8,9]$.

\section{Results of the monitoring method}

In the context of train maintenance, the MCT method is used to simultaneously identify the six suspension parameters of interest using all the available data. A single likelihood function is thus computed from the measurements of the four acceleration signals, on all the available track stretches. This likelihood function takes as argument the six-dimensional vector of parameters. The MCT method is tested on different cases. Section 6.1 explains how numerical experiments are used to validate the method. Section 6.2 compares the $\mathrm{KP}$ and MCT methods on both numerical experiments and real measurements. Section 6.3 studies the long-term evolution of the suspension mechanical parameter by applying the MCT method on real measurements obtained at several different dates. They are denoted $\left(T_{k}\right)_{k}$ with $k=-5 \ldots 4$ and chronologically 
ordered. The time steps between dates are of several months. Date $T_{0}$ corresponds to the reference date, used to identify error $\mathbf{B}$, for which the parameters are supposed to be nominal. A major maintenance operation took place between $T_{-1}$ and $T_{0}$, during which all the considered suspension elements have been replaced.

For each date, measurements of the track geometry and of the train dynamic behaviour on an entire TGV line are used. In addition, we considered measurements with the equipped train traveling in both orientations, that is to say with the equipped bogie located at the head and at the rear of the train. This adds up to around 800 kilometers of measurements, which represent around 100 individual Vampire simulations taking a few minutes each (but that have been performed using parallel computing).

In order to build the surrogate model of the likelihood function, these Vampire simulations have been performed 500 times with different values of the suspension parameters. The latter are scattered evenly in the six-dimensional admissible set of the parameters that consists of the set product of the admissible intervals of each parameters. For a given parameter, the admissible interval encloses the expected possible values of the parameter for a degraded suspension element. The interval is centered on the nominal parameter value. Its width has been determined using the train building specifications. No statistics were actually available to better characterize these admissible intervals, coming from bench tests of discarded suspension elements for instance.

Remark. For a complete validation of the proposed suspension monitoring method, field tests would be necessary. They would consist in different steps:

- bench-test degraded suspension elements in order to characterize their mechanical parameters;

- equip them on a train and measure the in-line dynamic behaviour of the train thanks to the accelerometers layout described in Section 3;

- perform the identification using these measurements;

- compare the identified parameters values to the values obtained from the bench tests.

For cost and complexity reasons, such a test campaign has not been carried out yet (note that for a contribution on this topic, the reader can be referred, for instance, to [28])

\subsection{Method validation with numerical experiments}

In the present case, a numerical experiment consists of simulated train responses that are used as if they were experimental data. They are generated using actual measurements of the track geometry irregularities on several track stretches and known degraded suspension parameters. Moreover, an independent realisation of the error $\mathbf{B}$ is added to the response signal on each track stretch in order to generate a quantity as close as possible to an actual measurement. The numerical experiments allows for validating the calibration procedure: the procedure is applied on the virtual train response, the calibration results can then be compared to the reference parameters used to generate the response.

We suppose that a set of $\nu_{1}$ track irregularities measurements $\left\{\mathbf{x}^{\text {mes }, i}\right\}_{1 \leq i \leq \nu_{1}}$ is available. The validation procedure from a numerical experiment can then be summed up as follows. 
(1) Choose vector $\mathbf{w}_{1}$ of parameters;

(2) Run the simulation on the $\nu_{1}$ track stretches with $\mathbf{w}_{1}$;

(3) Generate $\nu_{1}$ independent realisations $\left\{\mathbf{b}^{i}\right\}_{1 \leq i \leq \nu_{1}}$ of $\mathbf{B}$;

(4) Add these realisations to the simulated response to obtain realisations of the train dynamic response

$$
\mathbf{y}^{\text {num }, i}=\mathbf{h}\left(\mathbf{x}^{\text {mes, } i} ; \mathbf{w}_{1}\right)+\mathbf{b}^{i}, 1 \leq i \leq \nu_{1} ;
$$

(5) Perform the calibration using input data

$$
\left\{\left(\mathbf{x}^{\mathrm{mes}, i}, \mathbf{y}^{\mathrm{num}, i}\right)\right\}_{1 \leq i \leq \nu_{1}}
$$

to obtain the calibrated random vector $\mathbf{W}_{1}^{\text {post }}$

(6) Compare the samples of $\mathbf{W}_{1}^{\text {post }}$ to $\mathbf{w}_{1}$.

Figure 7 sums up the calibration results in three cases. For the first one, the chosen parameters are set to the nominal, $\mathbf{w}_{1}=\mathbf{w}_{0}$. For the other two, their are arbitrarily chosen in the admissible set $\mathcal{C}_{\mathbf{W}}$. For each case a good correspondence is obtained between the calibrated mean value $E\left\{\mathbf{W}_{1}^{\text {post }}\right\}$ and the chosen parameter $\mathbf{w}_{1}$. The error is always below $5 \%$ of the size of the admissible interval for each parameter. The standard deviations of the calibrated parameters are small, which indicates a high confidence in the identified parameter values. This confidence results from the large quantity of data that is used to perform the identification. They may vary from one parameter to another one. The first explanation is the sensitivity of the train response to the different parameters. Since the results are scaled, these differences also depend on the initial choice for the admissible interval size.

\subsection{Comparison of the KP and MCT method}

Figure 8 compares the KP and MCT methods, on a numerical experiments and using actual acceleration measurements (at date $T_{1}$ ). Concerning the calibrated mean values, no difference is observable between the two methods. However, the calibrated standard deviations are significantly smaller with the KP method. This is coherent with the fact that it does not take into account the surrogate model uncertainty. We can conclude that the KP method tends to overestimate the confidence in the identified values of the parameters. It can be assumed that the level of confidence obtained with the KP method is comparable to the one that would be obtained if no surrogate model was used.

It should also be noted that the standard deviations are larger for the parameter identification performed on the real measurements than on the numerical experiments. This result seems to indicate that the output predictive error $\mathbf{B}$ does not perfectly represent the distance between the measured and simulated train responses. Two main reasons can explain that: the Gaussian assumption for error $\mathbf{B}$, and the limited number of realisations used to identify $\mathbf{B}$ compared to its dimension.

\subsection{Study of the parameter evolution from experimental data}

Figures 9 and 10 present the time evolution of the six suspension parameters of interest as identified using the MCT method from the measurements performed at the ten dates $\left(T_{k}\right)_{k}$. It has to be noted that for the dates before maintenance $\left(T_{-5}, \ldots T_{-1}\right)$, it 

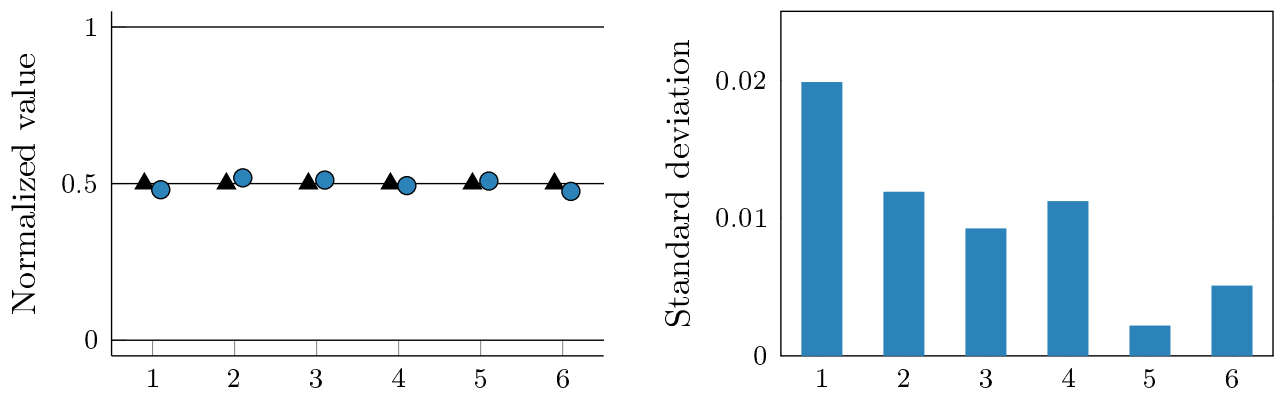

(a)
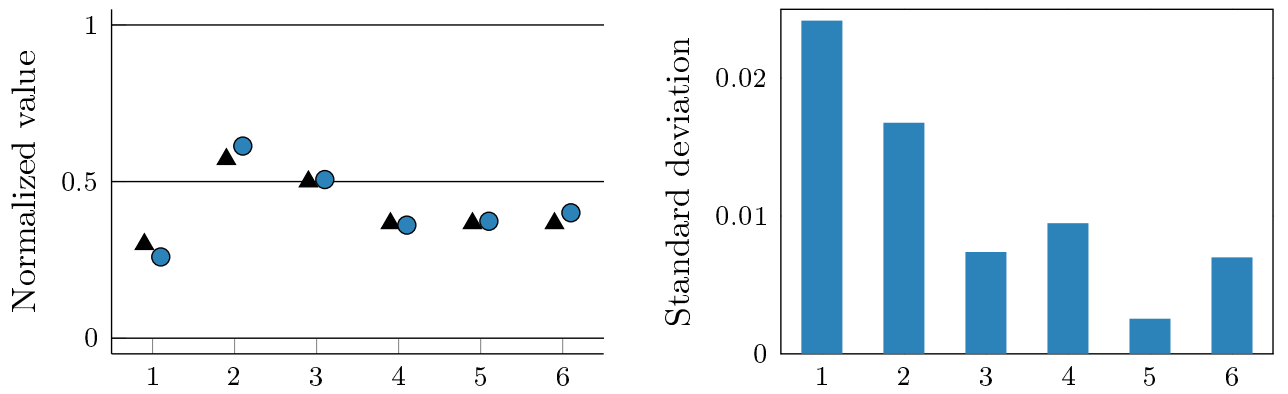

(b)
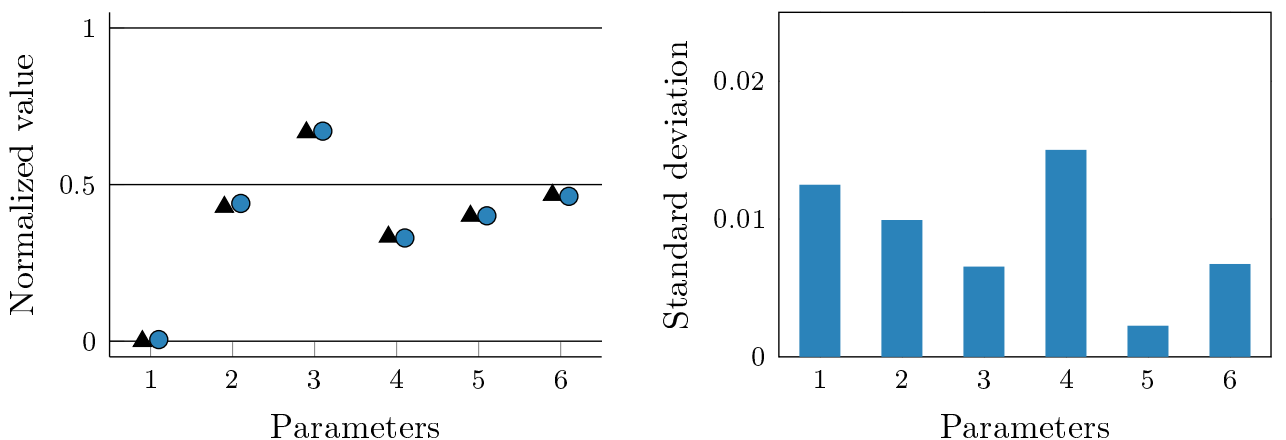

(c)

Figure 7. Numerical experiments results (using the MCT method). The left graphs compare calibrated mean values $E\left\{\mathbf{W}_{1}^{\text {post }}\right\}$ (blue dots) to the chosen parameters $\mathbf{w}_{1}$ (black triangles). The scale is normalized, meaning that the admissible interval has been shrunk to $[0,1]$ for each parameter ( 0.5 is then the nominal value). The right graphs present the standard deviations of $\mathbf{W}_{1}^{\text {post }}$, on the same normalized scale. In case (a), the parameter values are set to the nominal. They are arbitrarily chosen for case (b) and (c). 

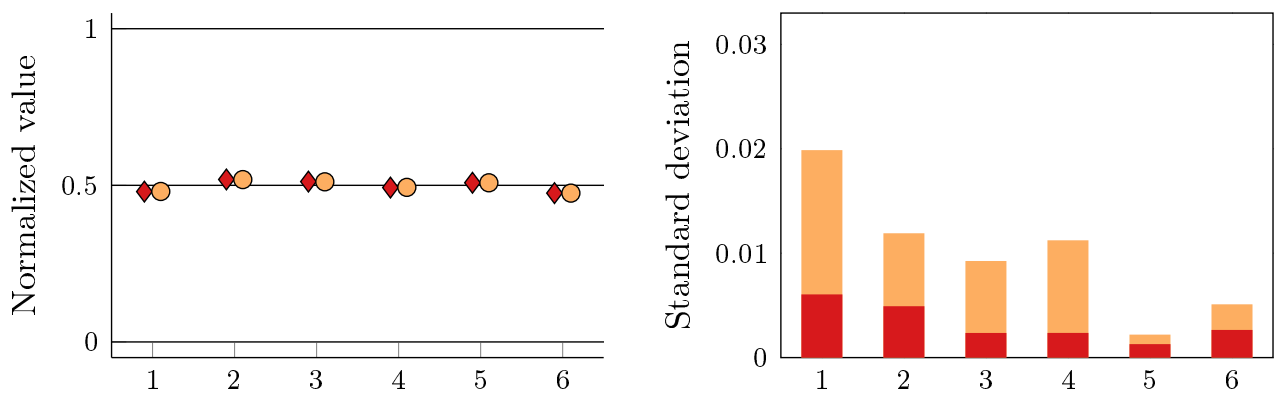

(a)
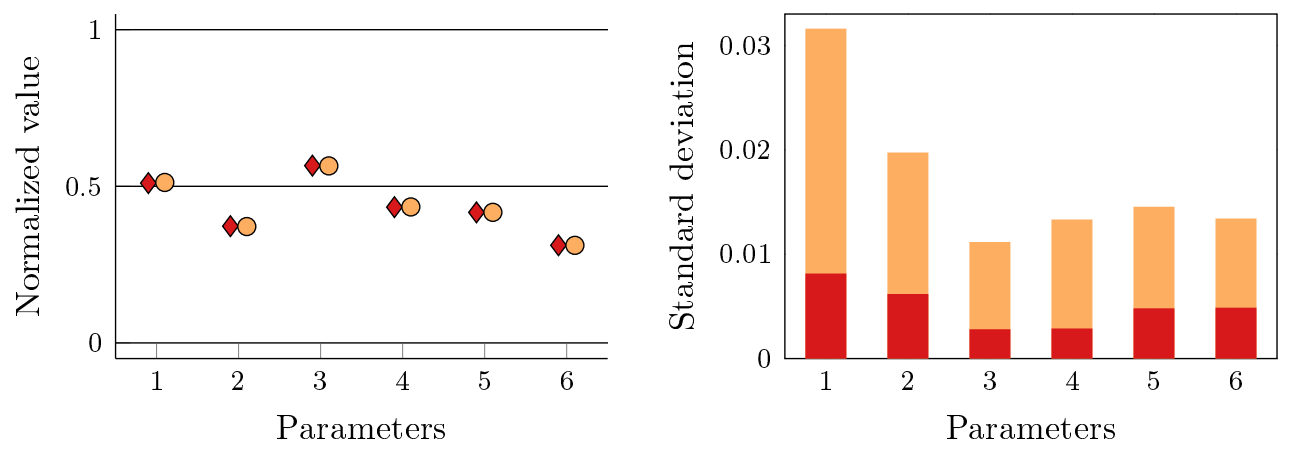

(b)

Figure 8. Comparison of the KP and MCT method. The left graphs compare the calibrated mean values of the parameters using the KP (red diamonds) and the MCT (orange dots) methods. The axes layout is identical to Figure 7. The right graphs compare the calibrated standard deviations of the parameters using the KP (red or dark gray) and the MCT (orange or light gray) methods. Case (a) corresponds to the numerical experiment with parameter values set to the nominal, while case (b) corresponds to a parameter identification using the real measurements at date $T_{1}$ 

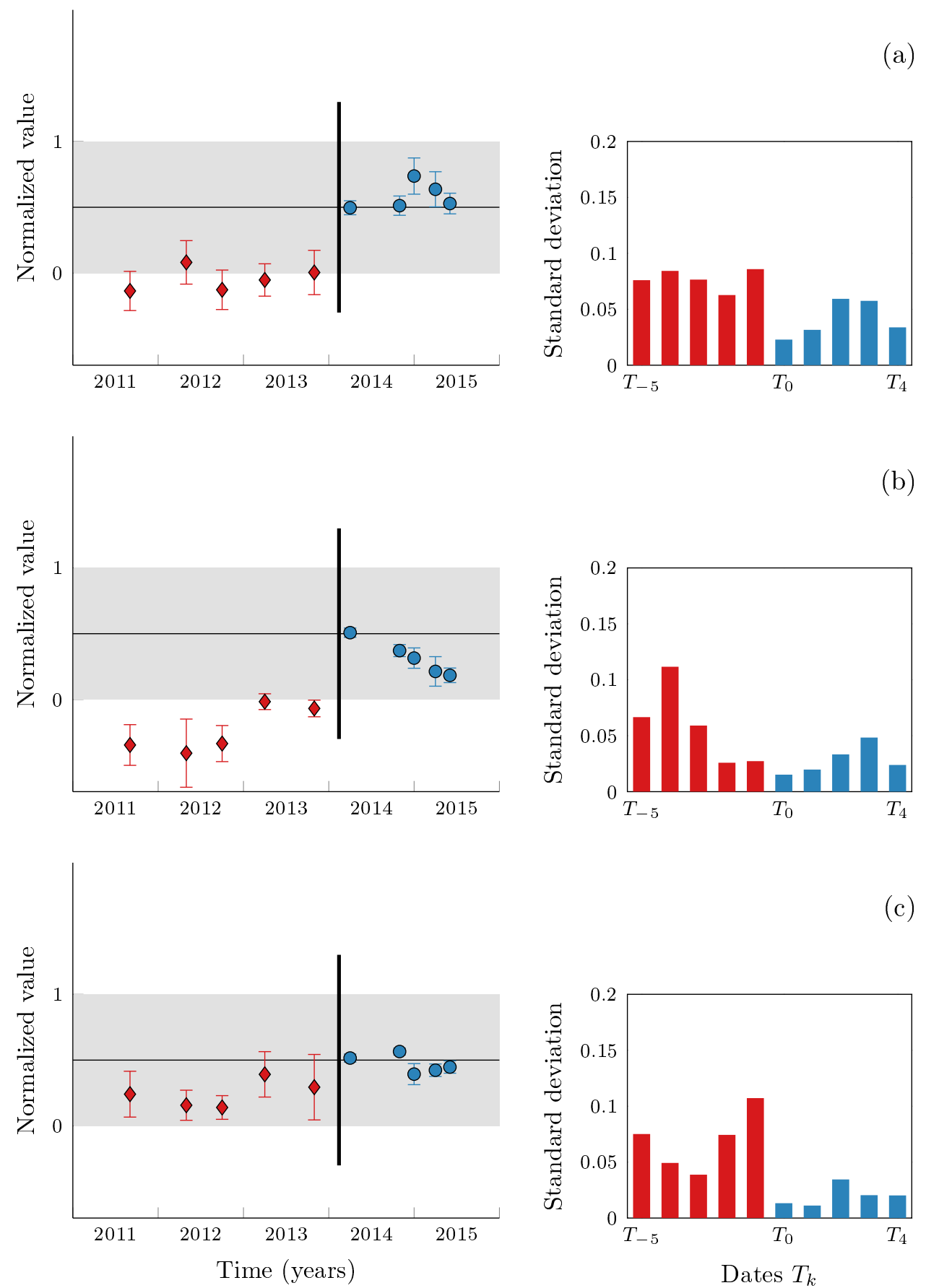

(b)

(a)

$T_{4}$

(c)

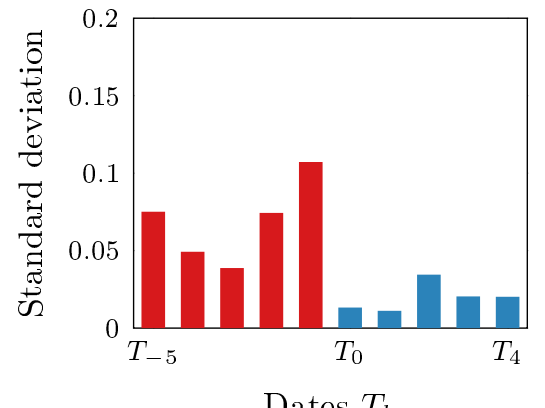

Figure 9. Parameter identification at ten dates $T_{k}$ for three mechanical parameters: (a) Airspring stiffness, (b) Airspring damping coefficient, (c) Primary elastomeric stiffness. The left graphs present the time evolution of calibrated mean value of the parameter, surrounded by the $98 \%$ confidence interval. The red diamonds indicate the dates before maintenance (represented by the vertical line), the blue dots the dates after. The scale is normalized, meaning that the (initial) admissible interval has been shrunk to $[0,1]$ for each parameter $(0.5$ is then the nominal value). The right graphs present the time evolution of the calibrated standard deviation, on the same normalized scale. 


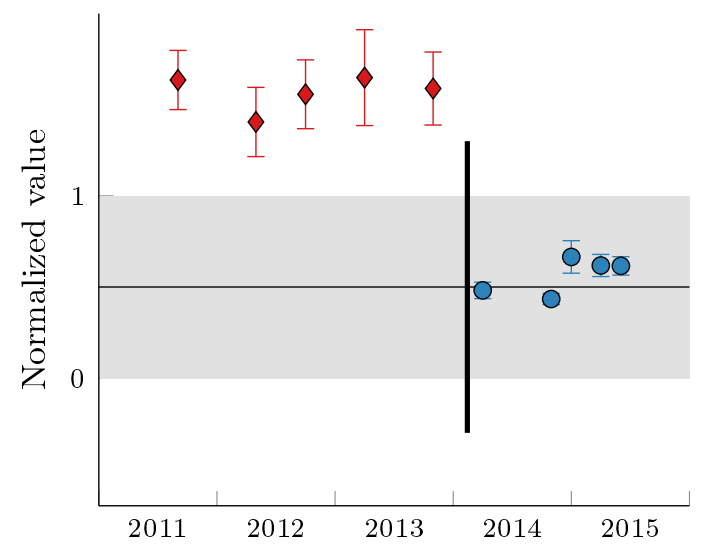

(d)
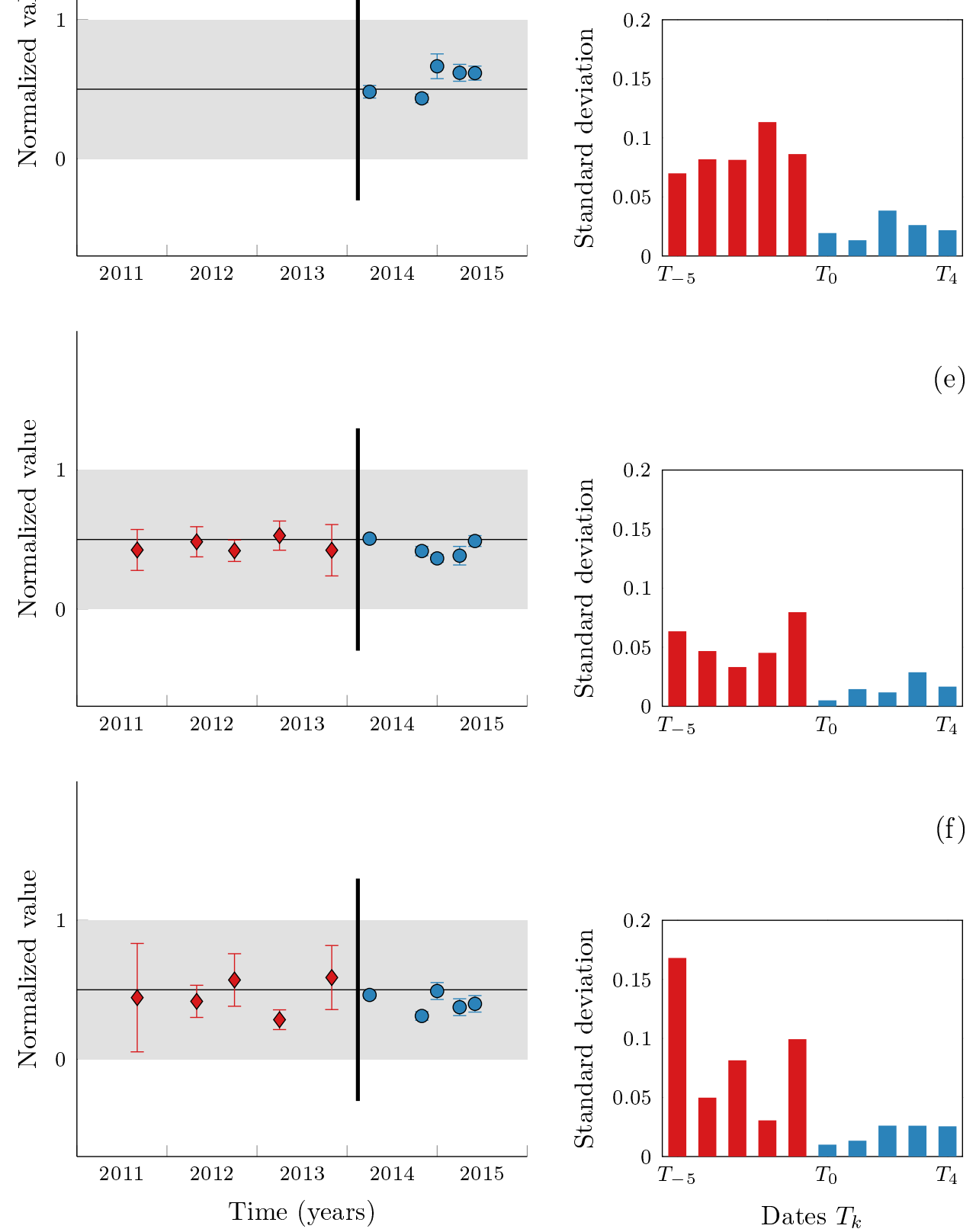

Figure 10. Parameter identification at ten dates $T_{k}$ for three mechanical parameters: (d) Primary vertical damper, (e) Yaw damper, (f) Upper inter-trailer damper. The axes layout is identical to Figure 9. 
was necessary to enlarge certain parameters admissible intervals, or else the identified parameters would remain stuck on the boundaries. However, in the graphs, the normalisation remains unchanged: the interval $[0,1]$ still corresponds to the initial admissible intervals.

We can first observe that the calibrated parameters at reference date $T_{0}$ (first blue dot) are close to the nominal values. This is in accordance to the assumption made to identify the output predictive error $\mathbf{B}$, and constitutes a second type of validation of the method.

Important difference can be noted between the results before and after the maintenance. The identified parameters are further from the nominal before maintenance, except for the yaw and inter-trailer dampers, for which the values are similar before and after maintenance. It corresponds to the fact that the suspension elements are more degraded before the maintenance operation. The confidence intervals (whose size is proportional to the identified parameter standard deviation) are much wider before maintenance. An explanation is the fact that the error $\mathbf{B}$ represents better the distance between the measured and simulated responses when the parameters are close to their nominal values. We can observe that the time evolution of the parameters identified by the MCT method is generally non-monotonic, which is unexpected.

The values identified for the primary vertical dampers, on graph (d), seem unrealistic before maintenance. When degraded, the dampers usually witness a decrease of their mechanical characteristics, while the identification results show a large increase with respect to the nominal. The analysis of the measured train dynamic response suggests that non-linearities affect the primary suspension, but are not accounted for in the train model. The identified parameter values for the primary vertical dampers may result from an attempt of the calibration procedure to compensate an evolution of these non-linearities.

\section{Conclusion and perspectives}

In this paper, we have proposed a novel model-based monitoring method for high-speed train passive suspensions. The main advantages are its ability to achieve a simultaneous identification of the suspension parameters to cope with possible couplings, to take into account the system uncertainties to perform a robust calibration, and to exploit a large number of complex measurements consisting of acceleration signals in the bogies and carbodies. The problem consists in an inverse parameter identification problem with uncertainties that we chose to solve in a Bayesian framework. Because the method relies on an expensive simulation code, we face a numerical cost issue that is addressed thanks to a Gaussian process surrogate model. The latter is used to efficiently approximate the likelihood function that is at the core of Bayesian approaches. However, using a surrogate model introduces a new type of uncertainty in the identification procedure. We propose a solution to take the latter into account thanks to a Monte Carlo sampling of trajectories of the likelihood surrogate model.

The method is validated on numerical experiments with a virtual train. The results show that the proposed novel approach is efficient. They also highlight the importance of taking into account the surrogate model uncertainty to correctly estimate the confidence intervals. The method is eventually applied on the measurements performed at several dates (with a time step of several months) in order to study the degradation over time of the suspension mechanical parameters. Although a clear effect of the maintenance can be observed, the evolution of the identified parameters appears 
non-monotonic. Consequently, the method has shown its ability to detect evolutions of the health state of suspension elements. These last results also suggest that further work on the train model is necessary: certain non-linearities are not accounted for and improvements of the suspension parametrisation are needed. More complex degradation patterns could also provide more accurate results. These last considerations are driving our current research.

\section{Acknowledgments}

This research has been supported by $S N C F$, the French National Railway Company.

\section{References}

[1] Li C, Luo S, Cole C, et al. An overview: modern techniques for railway vehicle on-board health monitoring systems. Vehicle System Dynamics. 2017;55(7):1045-1070.

[2] Perrin G, Duhamel D, Soize C, et al. Quantification of the influence of the track geometry variability on the train dynamics. Mechanical Systems and Signal Processing. 2015; 60:945-957.

[3] Lestoille N, Soize C, Funfschilling C. Sensitivity of train stochastic dynamics to long-time evolution of track irregularities. Vehicle System Dynamics. 2016;54(5):545-567.

[4] Lestoille N, Soize C, Funfschilling C. Stochastic prediction of high-speed train dynamics to long-term evolution of track irregularities. Mechanics Research Communications. 2016; 75:29-39.

[5] Hung CF, Hsu WL. Influence of the long-wavelength track irregularities on the motion of a high-speed train. Vehicle System Dynamics. 2018;56:95-112.

[6] Bing AJ, Gross A. Development of railroad track degradation models. Transportation research record. 1983;939:27-31.

[7] Weston P, Roberts C, Yeo G, et al. Perspectives on railway track geometry condition monitoring from in-service railway vehicles. Vehicle System Dynamics. 2015;53(7):10631091.

[8] Lebel D, Soize C, Funfschilling C, et al. Statistical inverse identification for nonlinear train dynamics using a surrogate model in a bayesian framework. Journal of Sound and Vibration. Submitted June 2018;

[9] Lebel D. Statistical inverse problem in nonlinear high-speed train dynamics [dissertation]. Université Paris-Est, Marne-la-Vallée; 2018.

[10] Sunder R, Kolbasseff A, Kieninger K, et al. Operational Experiences with Onboard Diagnosis System for High Speed Trains. In: Proceedings of the World Congress on Rail Research; 2001.

[11] Wei X, Jia L, Liu H. A comparative study on fault detection methods of rail vehicle suspension systems based on acceleration measurements. Vehicle System Dynamics. 2013; 51(5):700-720.

[12] Mei TX, Ding XJ. Condition monitoring of rail vehicle suspensions based on changes in system dynamic interactions. Vehicle System Dynamics. 2009;47(9):1167-1181.

[13] Martinod RM, Betancur GR, Heredia LFC. Identification of the technical state of suspension elements in railway systems. Vehicle System Dynamics. 2012;50(7):1121-1135.

[14] Gasparetto L, Alfi S, Bruni S. Data-driven condition-based monitoring of high-speed railway bogies. International Journal of Rail Transportation. 2013;1(1-2):42-56.

[15] Sakellariou JS, Petsounis Ka, Fassois SD. On board fault detection and identification in railway vehicle suspensions via a functional model based method. In: Proc. of ISMA, Leuven, Belgium; 2002.

[16] Alfi S, Bionda S, Bruni S, et al. Condition monitoring of suspension components in railway 
bogies. In: 5th IET Conference on Railway Condition Monitoring and Non-Destructive Testing (RCM 2011); 2011.

[17] Jesussek M, Ellermann K. Fault detection and isolation for a full-scale railway vehicle suspension with multiple Kalman filters. Vehicle System Dynamics. 2014;52(12):16951715 .

[18] Hayashi Y, Tsunashima H, Marumo Y. Fault Detection of Railway Vehicle Suspension Systems Using Multiple-Model Approach. Journal of Mechanical Systems for Transportation and Logistics. 2008;1(1):88-99.

[19] Mori H, Tsunashima H. Condition Monitoring of Railway Vehicle Suspension Using Multiple Model Approach. Journal of Mechanical Systems for Transportation and Logistics. 2010;3(1):243-258.

[20] Li P, Goodall R, Kadirkamanathan V. Estimation of parameters in a linear state space model using a Rao-Blackwellised particle filter. In: IEE Proceedings-control theory and applications; Vol. 151; 2004. p. 727-738.

[21] Li P, Goodall R, Weston P, et al. Estimation of railway vehicle suspension parameters for condition monitoring. Control Engineering Practice. 2007;15:43-55.

[22] Jesussek M, Ellermann K. Fault detection and isolation for a nonlinear railway vehicle suspension with a hybrid extended kalman filter. Vehicle System Dynamics. 2013; 51(10):1489-1501.

[23] Li P, Goodall R. Model-Based Condition Monitoring for Railway Vehicle Systems. In: Control 2004; 2004. September.

[24] Liu XY, Alfi S, Bruni S. An efficient recursive least square-based condition monitoring approach for a rail vehicle suspension system. Vehicle System Dynamics. 2016;54(6):814830.

[25] Kraft S. Parameter identification for a TGV model [dissertation]. Ecole Centrale Paris; 2012.

[26] Perrin G, Soize C, Duhamel D, et al. Track irregularities stochastic modeling. Probabilistic Engineering Mechanics. 2013;34:123-130.

[27] Perrin G, Soize C, Ouhbi N. Data-driven kernel representations for sampling with an unknown block dependence structure under correlation constraints. Computational Statistics and Data Analysis. 2018;119:139-154.

[28] Götz G, Polach O. Verification and validation of simulations in a rail vehicle certification context. International Journal of Rail Transportation. 2018;6(2):83-100. 